\title{
Quantifying collagen fiber orientation in breast cancer using quantitative phase imaging
}

\author{
Hassaan Majeed \\ Chukwuemeka Okoro \\ André Kajdacsy-Balla \\ Kimani C. Toussaint, Jr. \\ Gabriel Popescu
}




\title{
Quantifying collagen fiber orientation in breast cancer using quantitative phase imaging
}

\author{
Hassaan Majeed,,$^{a, \dagger}$ Chukwuemeka Okoro, ${ }^{b, \dagger}$ André Kajdacsy-Balla, ${ }^{c}$ Kimani C. Toussaint Jr., ${ }^{d}$ and \\ Gabriel Popescue,* \\ aUniversity of Illinois at Urbana Champaign, Quantitative Light Imaging (QLI) Lab, Department of Bioengineering, Beckman Institute of Advanced \\ Science and Technology, Urbana, Illinois, United States \\ bUniversity of Illinois at Urbana Champaign, Photonics Research of Bio/Nano Environments (PROBE) Lab, Department of Electrical and Computer \\ Engineering, Mechanical Engineering Lab, Urbana, Illinois, United States \\ 'University of Illinois at Chicago, Department of Pathology, Chicago, Illinois, United States \\ dUniversity of Illinois at Urbana Champaign, Photonics Research of Bio/Nano Environments (PROBE) Lab, Department of Mechanical Science and \\ Engineering, Mechanical Engineering Lab, Urbana, Illinois, United States \\ eUniversity of Illinois at Urbana Champaign, Quantitative Light Imaging (QLI) Lab, Department of Electrical and Computer Engineering, Beckman \\ Institute of Advanced Science and Technology, Urbana, Illinois, United States
}

\begin{abstract}
Tumor progression in breast cancer is significantly influenced by its interaction with the surrounding stromal tissue. Specifically, the composition, orientation, and alignment of collagen fibers in tumor-adjacent stroma affect tumor growth and metastasis. Most of the work done on measuring this prognostic marker has involved imaging of collagen fibers using second-harmonic generation microscopy (SHGM), which provides label-free specificity. Here, we show that spatial light interference microscopy (SLIM), a label-free quantitative phase imaging technique, is able to provide information on collagen-fiber orientation that is comparable to that provided by SHGM. Due to its wide-field geometry, the throughput of the SLIM system is much higher than that of SHGM and, because of the linear imaging, the equipment is simpler and significantly less expensive. Our results indicate that SLIM images can be used to extract important prognostic information from collagen fibers in breast tissue, potentially providing a convenient high throughput clinical tool for assessing patient prognosis. $\odot 2017$ Society of Photo-Optical Instrumentation Engineers (SPIE) [DOI: 10.1117/1.JBO.22.4.046004]
\end{abstract}

Keywords: breast cancer; quantitative phase imaging; second-harmonic generation microscopy; cancer prognosis; histopathology. Paper 160836RR received Dec. 9, 2016; accepted for publication Mar. 16, 2017; published online Apr. 7, 2017.

\section{Introduction}

With 522,000 reported deaths worldwide in 2012, breast cancer is the most prevalent type of cancer among women and the second most prevalent cancer overall according to the World Health Organization (WHO). ${ }^{1,2}$ The WHO has also emphasized the importance of early diagnosis and prognosis in improving health outcomes. This need has led to significant research into discovering new biomarkers for assessing a patient's health status early on. ${ }^{3-5}$ Current markers, such as histological grade, hormone receptor status, tumor size, etc., while useful for most patients, do not accurately predict outcomes for all patients. Thus, there is a need to expand on the current prognostic markers to account for biological variation among individuals. ${ }^{4,6}$ Furthermore, reliable prognostic markers help clinicians predict the potential for metastasis and avoid overtreatment. ${ }^{6}$

The role of adjacent stroma in mediating breast tumor initiation, progression, and invasion to surrounding tissue has been extensively discussed over the years. ${ }^{7-11}$ Tumor invasion into surrounding healthy tissue involves breaking down of the basement membrane and a desmoplastic response in the stroma. This response involves an increase in density of the extracellular matrix, marked by increased deposition of collagen, as well as recruitment of stromal cells (e.g., fibroblasts and inflammatory cells) to facilitate tumor growth. ${ }^{4,8,9}$ Using both mouse and

*Address all correspondence to: Gabriel Popescu, E-mail: gpopescu@illinois .edu

†These two authors have made equal contributions to this work. in vitro models of mammary tissue, studies have also concluded that tumor progression is marked by realignment and reorientation of collagen fibers. ${ }^{4,12-14}$ For example, Conklin et al. ${ }^{4}$ showed that the tumor adjacent collagen signature 3 (TACS 3) correlates with lower disease-free and disease-specific survival in breast cancer patients. ${ }^{15}$

Second-harmonic generation microscopy (SHGM) has emerged as a powerful technique for imaging collagen fibers in breast cancer stroma with subcellular resolution. ${ }^{16}$ SHGM measurement of the prognostic signature TACS 3 was reported in Ref. 4. Ambekar et al. ${ }^{17}$ used Fourier analysis on SHGM images to show that the collagen fibers in breast biopsies are more aligned in malignant versus premalignant and benign tissue. Riching et al. ${ }^{18}$ used SHGM to image 3-D collagen gels to elucidate specific epithelial cell-fiber interactions that are responsible for enhancing tumor progression along aligned fibers in breast tissue. Other researchers have used image segmentation and machine learning tools to extract prognostic information from SHGM images of collagen fibers. ${ }^{19,20}$

SHGM maps the second-order nonlinear susceptibility $\chi^{(2)}$ associated with noncentrosymmetric molecules and thus probes fibrillar collagen structures with specificity. ${ }^{16}$ As detailed in Sec. 2, collagen generates a strong second-harmonic signal compared to the surrounding cellular structures, resulting in high imaging contrast. ${ }^{21}$ However, the low contrast of other cellular structures in SHGM images means that the tumor boundary is difficult to delineate and often other imaging modalities are

1083-3668/2017/\$25.00 @ 2017 SPIE 
required..$^{20}$ Furthermore, since SHGM systems employ a laser point-scanning geometry, the imaging throughput is low and whole slide scanning of breast cancer tissue sections remains a tedious task.

Recently, QPI ${ }^{22}$ has become an emerging tool for label-free mapping of the optical path-length difference across a sample, with applications in various areas of medicine, biology, and material science. ${ }^{22-33}$ In clinical applications, QPI has been extensively used for blood analysis ${ }^{24,25,29,34-40}$ as well as cancer diagnosis $^{41-47}$ and prognosis ${ }^{48,49}$ (for a recent review, see Ref. 50).

SLIM is a QPI technique characterized by diffraction-limited transverse resolution and optical-path length sensitivity of less than a nanometer. ${ }^{27,51,52}$ It has been employed in the past for diagnosis of breast cancer ${ }^{42}$ as well as diagnosis and prognosis of prostate cancer. ${ }^{5,41,49}$

In this paper, we show that SLIM, in conjunction with basic image segmentation, is able to provide information on collagenfiber orientation and alignment similar to that obtained using SHGM. In addition, SLIM generates contrast for epithelial cells as well, which can be used to detect tumor boundaries. Furthermore, compared to SHGM, the SLIM images are acquired at a much higher throughput due to the wide-field geometry. Recently, SLIM has been automated and developed into the first QPI tissue scanner. Using both SLIM and SHGM, we imaged a tissue microarray (TMA) of breast biopsy cores consisting of both benign cases and malignant cases at different stages of the disease. Using the Fourier analysis method described in Sec. 3, we show that both SLIM and SHGM images generate similar histograms of fiber orientation angle.

This manuscript is organized as follows. In Sec. 2, we present the theoretical foundation for extracting second-order nonlinear response from phase-resolved linear imaging. Section 3 describes the experimental details of the study, and Sec. 4 presents the results. Section 5 summarizes and discusses these results.

\section{Theory}

In this section, we explain the theoretical motivation for our SLIM phase imaging by showing that the signal measured in SLIM is related to that measured in SHGM. The source of the signal measured in both modalities is the induced polarization $\mathbf{P}$. To the second-order approximation, this can be related to the fundamental (incident) field $\mathbf{E}$ as

$\mathbf{P}=\chi^{(1)}(\omega) \mathbf{E}+\chi^{(2)}(2 \omega) \mathbf{E}^{2}$,

where $\chi^{(1)}$ is the first-order electric susceptibility, $\chi^{(2)}$ is the second-order nonlinear susceptibility, and $\omega$ is the optical frequency of the source. ${ }^{53}$

The physical quantity measured in SLIM is the spatially resolved linear response, $\chi^{(1)}(\mathbf{r})=n^{2}(\mathbf{r})-n_{0}^{2}$ with $n$ being the refractive index of tissue, $n_{0}$ being the refractive index of the immersion medium, and $\mathbf{r}=(x, y)$. Note that for low refractive index contrast, $\chi^{(1)}$ simplifies to $\chi^{(1)}(\mathbf{r}) \approx 2 n_{0}\left[n(\mathbf{r})-n_{0}\right]$. The SLIM signal is the optical path-length map generated by the tissue slice:

$\phi(\mathbf{r})=\beta_{0}\left[n(\mathbf{r})-n_{0}\right] t \quad \approx \beta_{0} \frac{\chi^{(1)}(\mathbf{r})}{2 n_{0}} t$,

where $\beta_{0}=2 \pi / \lambda$ is the wavenumber in vacuum, $t$ is the local thickness of tissue, and $\lambda$ is the wavelength of the illumination. ${ }^{22}$
The physical quantity measured in SHGM is $\chi^{(2)}(2 \omega)$, which relates to the SHG electric field $\mathbf{E}_{\mathrm{SHG}}(2 \omega)$ via ${ }^{16,53-55}$

$\mathbf{E}_{\mathrm{SHG}}(2 \omega) \propto \beta_{0}^{2} \chi^{(2)}(2 \omega) \mathbf{E}^{2}(\omega)$.

We can find a relationship between the $\chi^{(2)}(2 \omega)$ and $\chi^{(1)}(\omega)$ signals measured by our two imaging methods by solving the equation of motion for the anharmonic oscillator describing microscopic charge displacement (see, for example, Sec. 1.4. in Ref. 53). This leads to the expression:

$$
\left[\frac{2 n_{0}}{\beta_{0} t} \phi(\mathbf{r}, \omega)\right]^{2}=\left[\chi^{(1)}(\mathbf{r}, \omega)\right]^{2}=\frac{1}{A} \frac{\chi^{(2)}(\mathbf{r}, 2 \omega)}{\chi^{(1)}(\mathbf{r}, 2 \omega)} .
$$

In Eq. (4), $A=\frac{a \varepsilon_{0}^{2} m}{N^{2} e^{3}}$, where $a$ is a constant that depends on the mechanical properties of the anharmonic oscillator model, $N$ is the volume density of electric dipoles in the medium, and $e$ is the elementary charge. Equation (4) indicates that the secondharmonic response is proportional to the square of the linear response, which is measured directly by SLIM, namely,

$\chi^{(2)}(\mathbf{r}, 2 \omega) \sim \chi^{(1)}(\mathbf{r}, 2 \omega) \phi^{2}(\mathbf{r}, \omega)$.

This relationship can then be used to rewrite Eq. (1) in the form:

$\mathbf{P}=\chi^{(1)}(\omega) \mathbf{E}+\chi^{(1)}(2 \omega) \phi^{2}(\omega) \mathbf{E}^{2}$.

In Eq. (6), the linear term generates the SLIM signal and provides contrast in both centrosymmetric and noncentrosymmetric structures. The quadratic term accounts for the SHGM signal and generates contrast only in noncentrosymmetric structures. ${ }^{53}$ Since the quadratic term has a dependence on $\phi(x, y)$, we anticipate that, in noncentrosymmetric regions (collagen fibers), SLIM and SHGM images provide similar morphological information. However, before a comparison between the two can be made, the centrosymmetric information in SLIM (linear imaging) needs to be extracted out. In this work, we extract this information out using image processing techniques, as discussed in Sec. 4.

\section{Materials and Methods}

\subsection{Second-Harmonic Generation Microscopy and Spatial Light Interference Microscopy Imaging Systems}

The optical setups for the two imaging modalities are illustrated in Fig. 1. While both have been discussed in detail in previous publications, ${ }^{17,51,56}$ here, we briefly describe their operating principles.

SLIM is built as a module at the output port of a commercial phase contrast microscope (Zeiss Observer Z1). The conjugate image plane at the output port of the microscope is imaged onto a CCD camera using a $4 f$ lens system (comprising lenses $\mathrm{L}_{1}$ and $\mathrm{L}_{2}$ ). At the Fourier plane of the first lens $\mathrm{L}_{1}$, a spatial light modulator controls the phase of the unscattered portion of light with respect to that of the scattered portion in increments of $\pi / 2$. The camera acquires four intensity images at four different phase modulations $(0, \pi / 2, \pi,-\pi / 2)$ and, from these images, the phase of the imaging field $\phi(x, y)$ can be computed, as detailed in Ref. 51. Throughout our experiments, we used a $40 \times / 0.75$ NA phase contrast objective. Using a slide-scanning 
SLIM Optical Setup

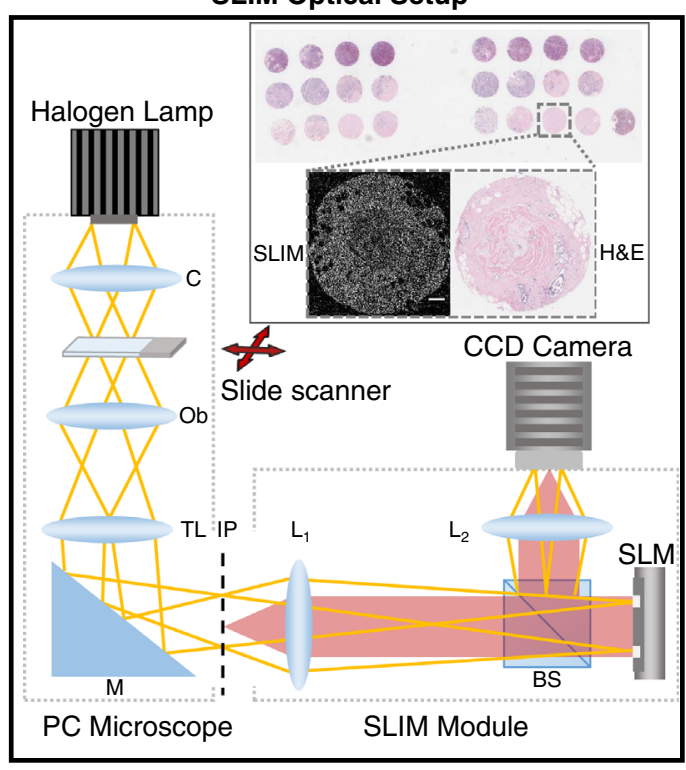

(a)
SHGM Optical Setup

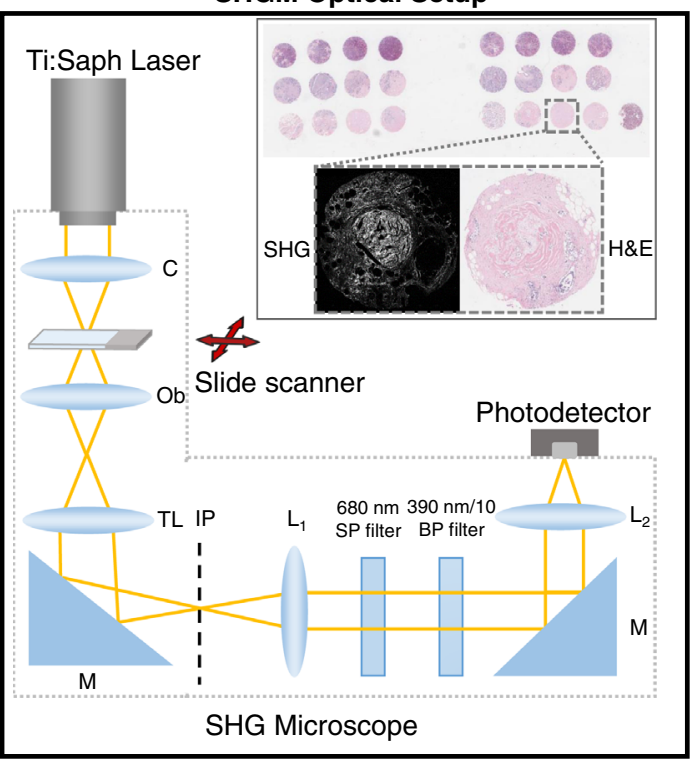

(b)

Fig. 1 (a) Optical setup of the SLIM system, built as a module attached to the output port of a commercial phase contrast microscope. The inset shows an H\&E image of the TMA slide, as well as H\&E and SLIM images of one of its cores. Scale bar: $200 \mu \mathrm{m}$. (b) Optical setup of the SHGM system. The inset shows an $\mathrm{H} \& \mathrm{E}$ image of the TMA slide as well as H\&E and SHGM images of one of its cores. C, condenser; Ob, objective; TL, tube lens; IP, image plane; M, mirror; BS, beam splitter; SP, short pass; BP, band pass; SLM, spatial light modulator.

platform, developed in-house using $\mathrm{C}++$, we imaged the entire TMA slide consisting of 24 cores at $0.5-\mu \mathrm{m}$ transverse resolution. Subsequent stitching of individual tiles and segmentation of individual core images were performed using algorithms developed in-house. ${ }^{42}$ The acquisition rate for the SLIM system was $0.08 \mu \mathrm{s} /$ pixel.

Figure 1(b) illustrates the SHGM system. A Ti:Sapphire laser was used to produce 70 -fs pulses at an excitation wavelength of $780 \mathrm{~nm}$ and a repetition rate of $80 \mathrm{MHz}$. The beam was scanned onto the sample and focused by a $20 \times / 0.8 \mathrm{NA}$ air-illumination Zeiss condenser. The transmitted beam was collected in the forward direction by a $40 \times / 0.9$ NA Zeiss objective and then passed through two filters. The first filter was a 680-nm short-pass filter $(680 \mathrm{~nm} / \mathrm{SP}-25)$ for blocking the laser light, and the second was a 390-nm band-pass filter $(390 \mathrm{~nm} \pm 18$ to $25 \mathrm{~nm}$ ) for selecting the second harmonic signal. In contrast to the wide-field CCD detector used in SLIM, SHGM uses a single-point photodetector. More details regarding the system have already been published in Refs. 17 and 56. The acquisition rate for SHGM system was $7 \mu \mathrm{s} /$ pixel.

\subsection{Tissue Microarray}

The breast TMA used in this study was purchased from US Biomax Inc. (Serial \# T088b). The TMA was received from the manufacturer with all human subject information deidentified. Neither the authors of this work nor their institutions were involved in tissue collection. The TMA is comprised of 24 cores from six different cases with eight benign/normal and 16 malignant cases. The malignant cases included cores corresponding to three different stages of breast cancer: IIa, IIb, and IIIa. The insets of both Figs. 1(a) and 1(b) show a hematoxylin and eosin (H\&E) stained bright-field image (henceforth referred to as "H\&E image") of the TMA. H\&E, SLIM, and SHGM images of one of the cores in the TMA are also shown.

Figure 2 shows H\&E, SLIM, and SHG images of three benign and three malignant cores, each at a different disease stage, as indicated. The SLIM images generate uniform contrast across the cores, including in cellular structures. SHGM images, on the other hand, generate contrast only in areas where collagen fibers are present. Note that the SHG signal is more sparse in the malignant cores versus the benign cores due to the higher fraction of epithelial cells in the former, associated with tumor invasion into surrounding stroma. Row 2 of Fig. 2 shows the square of the quantitative phase map $\phi(x, y)\left(\mathrm{SLIM}^{2}\right)$ for each core.

Figure 3 provides a comparison between morphologies of stromal and epithelial tissue within one core, as revealed in H\&E, SHGM, and SLIM images. As shown in Figs. 3(a) and $3(\mathrm{~g})$, both SLIM and SHGM reveal qualitatively similar stromal structures. The situation is different in areas with epithelial tissue, where SLIM images [Fig. 3(c)] contain centrosymmetric structures, such as epithelial cells, which are absent in SHGM images [Fig. 3(i)]. The presence of these cellular structures is confirmed by the H\&E stain, which shows cell nuclei in purple color [Fig. 3(1)]. In areas that are a mixture of cells and collagen fibers, quantifying fiber alignment and orientation from SLIM images requires segmentation of epithelial cells, as discussed in detail in Sec. 4.

\section{Results}

\subsection{Decoupling Isotropic from Anisotropic Signals in Spatial Light Interference Microscopy Images}

Prior to measuring collagen fiber orientation, the SLIM and SHGM images were registered and an image segmentation algorithm was used to remove isotropic structures from SLIM 


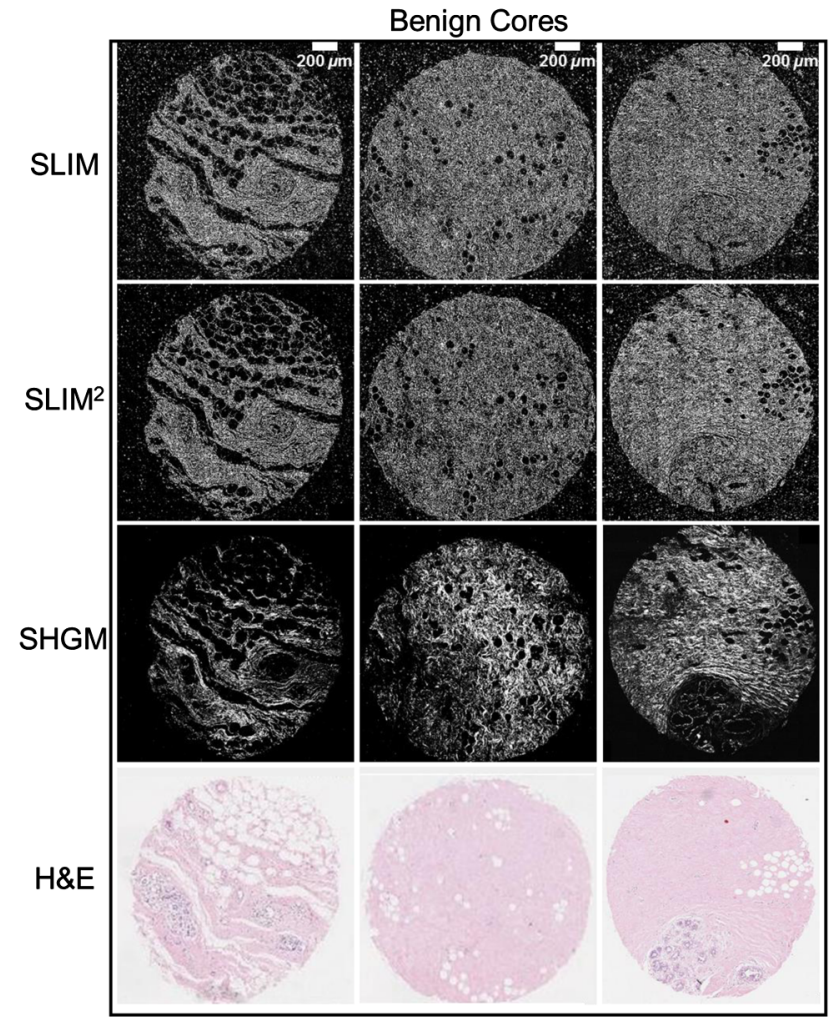

(a)

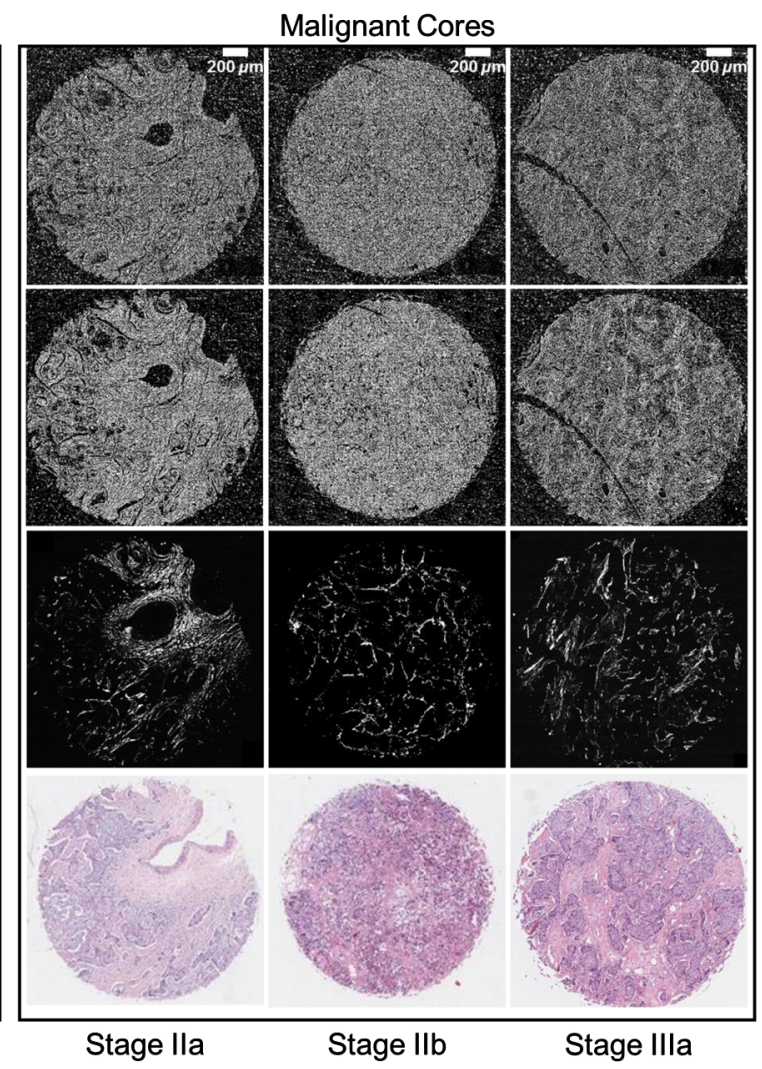

(b)

Fig. 2 Morphological comparison between (a) benign and (b) malignant cores from patients at three different stages of disease as captured by SLIM, SHGM, and H\&E images. The square of the quantitative phase map in SLIM is referred to as SLIM ${ }^{2}$ and is proportional to $\left[\chi^{(1)}\right]^{2}$.

images, leaving behind the highly anisotropic collagen fibers. No information from the SHGM data was used for the segmentation of SLIM images.

To perform image registration, the SLIM images were first downsampled, from $12,000 \times 12,000$ to $9216 \times 9216$ pixels/ core, to match the sampling of the SHGM images. These images were then coregistered using ImageJ by selecting control point pairs for each image and matching them using transformation techniques (primarily translation and rotation).

The image segmentation algorithm used for removing isotropic cellular structures from SLIM images is schematically illustrated in Fig. 4. Specifically, for each core image, we first computed the response to the Leung-Malik (LM) filter bank comprising gradient filters at 50 different orientations. ${ }^{57,58}$ Each filter in the bank computed the directional image gradient using the first derivative of a Gaussian, oriented at $\alpha=\frac{m \pi}{50}$, where $m=0,1,2, \ldots 49$, from the horizontal axis in the image. The resulting stack of responses represented both the magnitude and direction of the local gradient or anisotropy in a pixel's vicinity for each TMA core. The magnitude of these gradients was then normalized by the highest value in the stack and was summed along the stack (along the $z$-axis in Fig. 4). The resulting image represented the isotropy map of the tissue with higher values representing locally isotropic structures (background and cells) and lower values representing locally anisotropic structures (collagen fibers). This isotropy map was then low-pass filtered (Gaussian kernel of $15 \times 15$ pixels, which is slightly larger than one epithelial cell) and the Otsu's thresholding method was used to find the grayscale level separating anisotropic from isotropic pixels. ${ }^{59}$ The Otsu thresholding method dynamically sets the threshold by minimizing the intraclass variance and maximizing the interclass variance under the assumption of a biomodal class histogram distribution. Since this method establishes a threshold based on the natural separation of isotropic and anisotropic pixels in the data, it can be applied to arbitrary SLIM images without prior training. After setting the isotropic pixels to zero, we employed another round of low pass filtering (Gaussian kernel, $5 \times 5$ pixels) followed by thresholding to remove any remaining background pixels and obtain the label map. During this process, the maximum gradient along $z$ for each pixel was also used for detecting the remaining background pixels and marking them for removal. The final segmentation map was then computed using the label map as a mask. The segmentation algorithm was coded in MATLAB, and a prepublished LM filter generator was used for the purpose. ${ }^{60}$

As apparent from comparing the SLIM and segmented SLIM images in Figs. 4(f) and 4(g), the algorithm leads to oversegmentation, and sometimes collagen fibers that are isotropic at length scales equal to or smaller than the size of one epithelial cell are segmented out. In some cases, the segmentation algorithm may break up thin or twisting fibers. As shown in Sec. 4(c), these imperfections in segmentation are subdominant, and the local collagen orientation remains similar between SLIM and SHGM images. Furthermore, as demonstrated in Refs. 4, 20, and 55, biomarkers for prognosis are based on average orientation of collagen over spatial scales that are longer than those at which segmentation errors occur in our images. Thus, for the relevant clinical applications, sufficient information remains in the 
Stromal Tissue
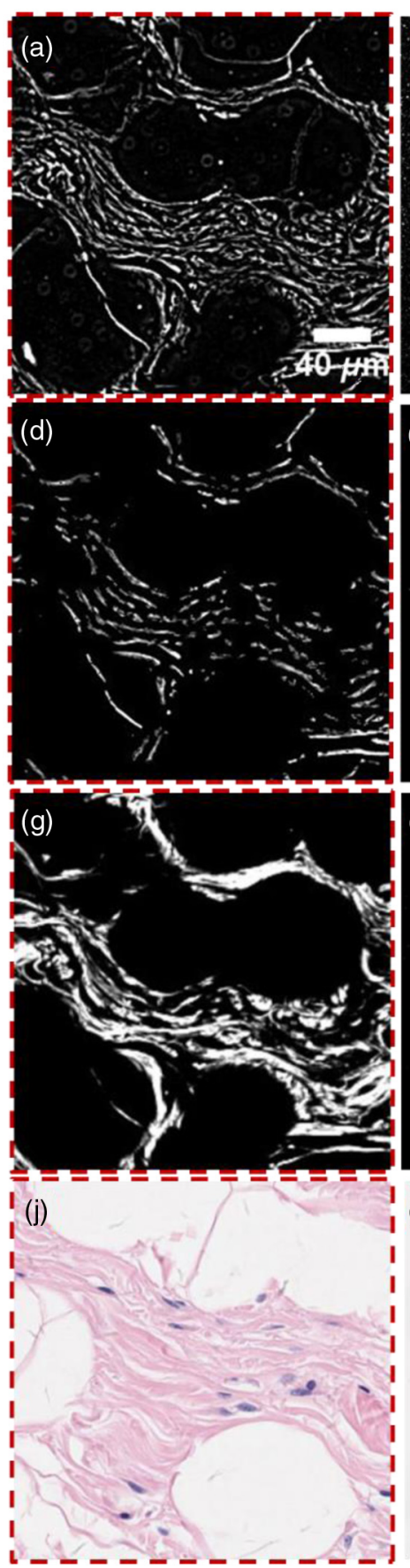

Stromal and Epithelial Tissue
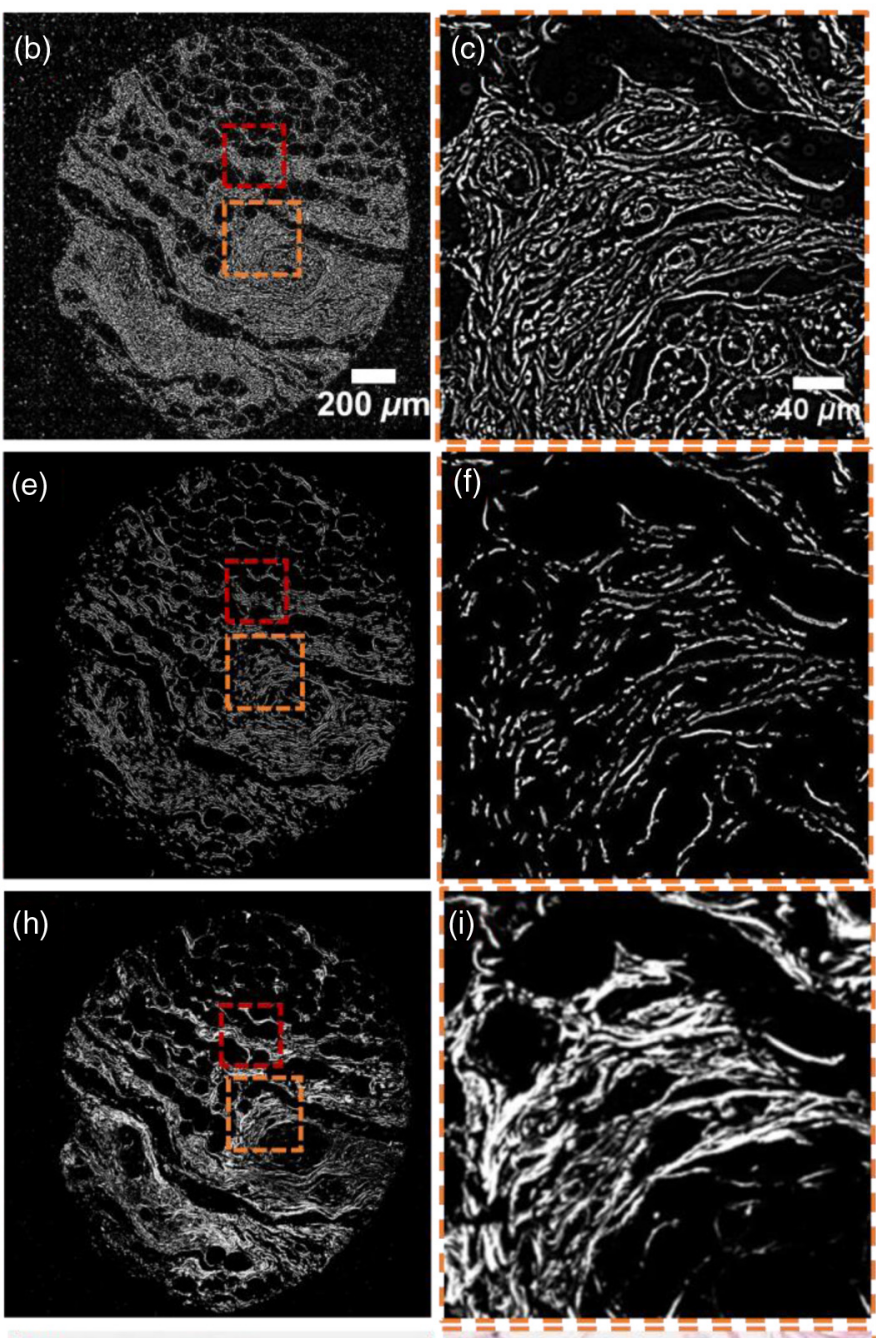

$(\mathrm{k})$
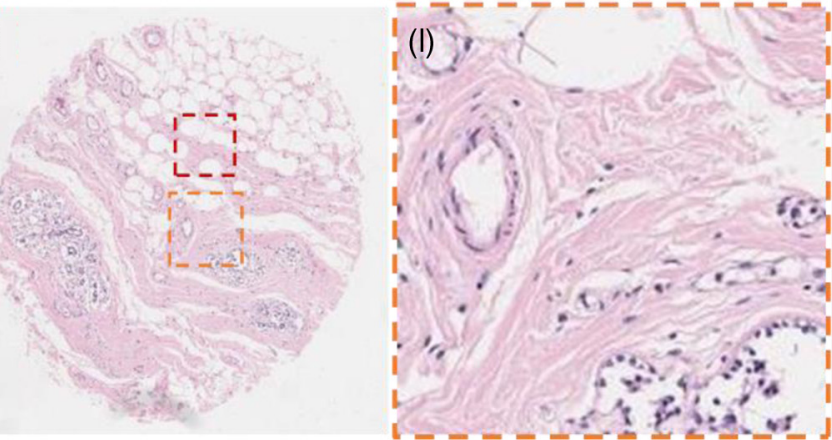

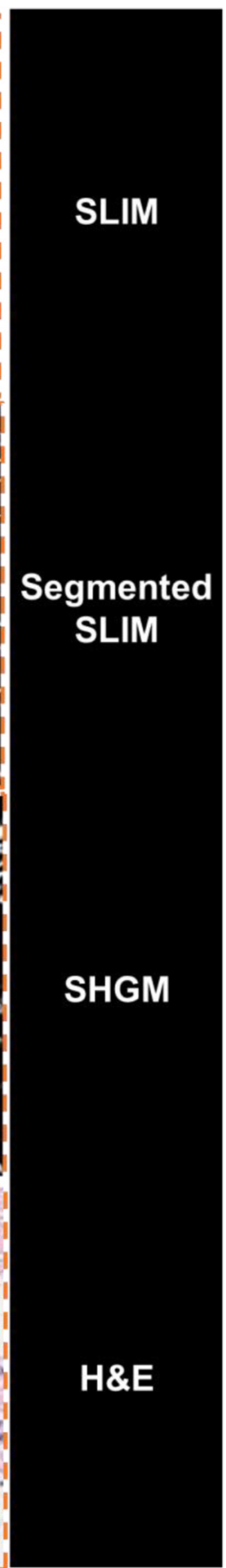

Fig. 3 (a-c) SLIM, (g-i) SHGM, and (j-l) H\&E images of both stromal and epithelial/stromal mixed tissue in a TMA core. $(a, d, g, j)$ Left-most and $(c, f, i, I)$ right-most columns show zoomed-in versions of the regions indicated on the core in the central column (b,e,h,k). As is evident from a comparison with the H\&E images, the SLIM images show contrast in both stromal and epithelial regions whereas the SHGM images only generate contrast in stromal regions. (d-f) Segmented SLIM images were obtained from the SLIM images by numerically removing the cellular structures and preserving the collagen fibers.

segmented SLIM images to measure fiber-orientation based biomarkers. Another important consideration for clinical applications is the relative orientation of tumor adjacent collagen fibers with the tumor boundary. While SLIM images can be segmented to digitally remove the epithelial cells, they clearly resolve epithelial structures, allowing determination of the tumor boundary orientation with respect to the collagen fibers.

\subsection{Fourier Analysis}

Fourier analysis was carried out on SHGM and segmented SLIM images to extract collagen fiber orientation. Each image was sectioned into subimage regions using grids $(16 \times 16$, $32 \times 32$, or $64 \times 64)$, and the localized orientation per subimage (which we call $\theta$ ) was determined using the Fourier analysis technique outlined in Ref. 55. Regions having a mean direction 


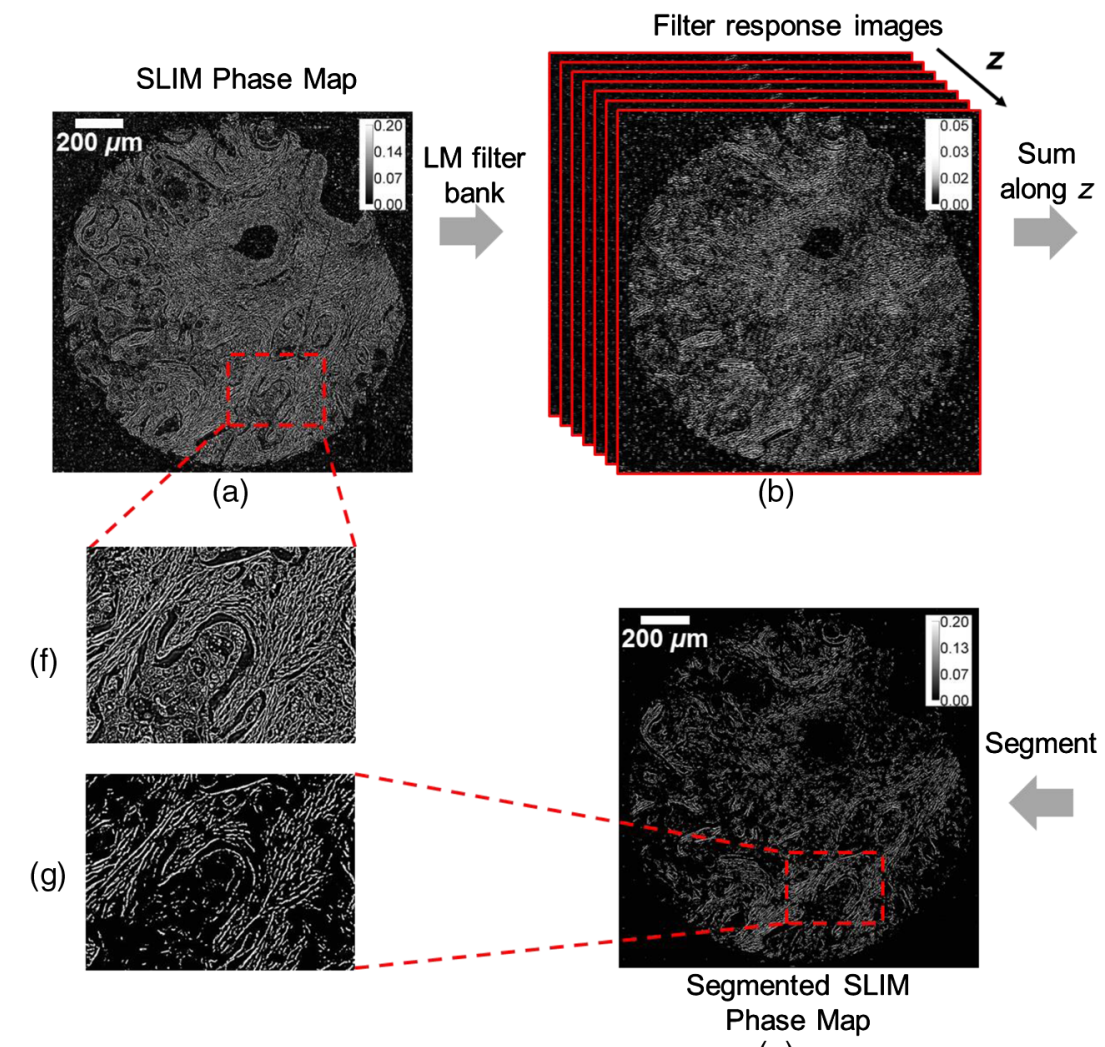

(e)

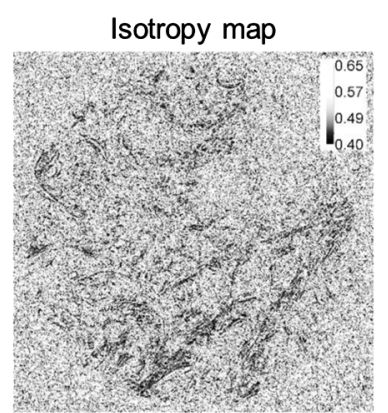

(c)

Threshold and label
Collagen pixels

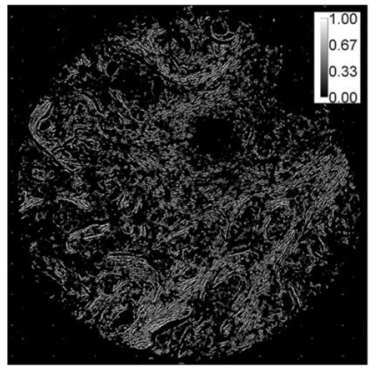

Label map

(d)

Fig. 4 The segmentation algorithm for removing isotropic structures from SLIM phase images. (a) SLIM phase map before segmentation (color bar in radians). (b) Response to LM filter bank. (c) Normalized isotropy map. (d) Binary label map. (e) Segmented SLIM image (color bar in radians). (f) and (g) Zoomedin portions of the SLIM and segmented SLIM images, respectively, showing an epithelial stromal boundary. The segmentation algorithm removes isotropic structures and preserves anisotropic signals associated with collagen fibers.

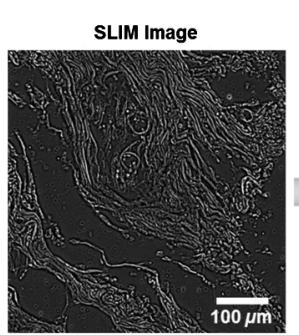

(a)

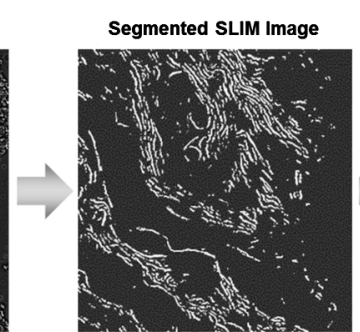

(b)

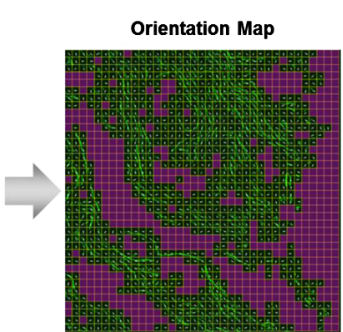

(c)

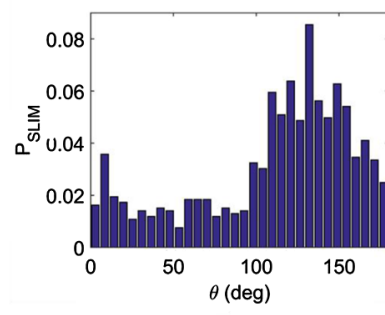

(f)

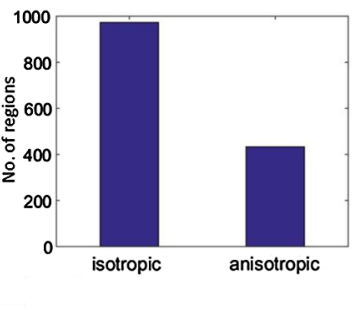

$\rho=0.86$

(g)

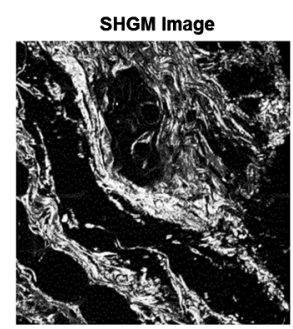

(d)

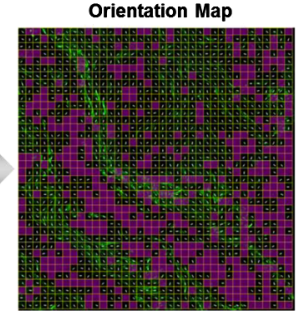

(e)

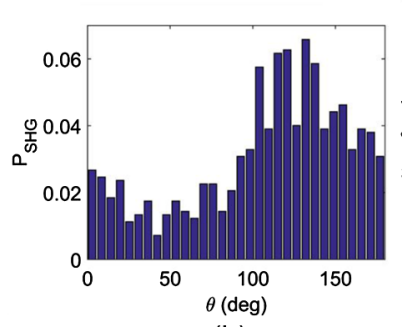

(h)

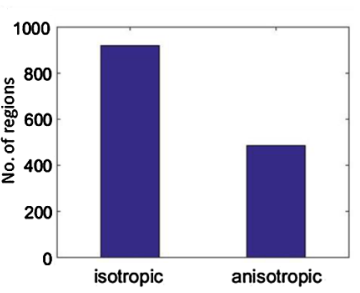

(i)

Fig. 5 The Fourier analysis procedure for computing collagen fiber orientation probability densities. (a) SLIM image. (b) Segmented SLIM image. (c) Orientation map of SLIM image. (d) SHGM image. (e) Orientation map of SHGM image. (f) Probability density of collagen fiber orientation $\theta$ computed from the SLIM orientation map in (c). (g) Bar chart showing the number of isotropic and anisotropic regions in segmented SLIM image. (h) Probability density of collagen fiber orientation $\theta$ computed from the SHGM orientation map in (e). (i) Bar chart showing the number of isotropic and anisotropic regions in SHGM image. The Pearson's correlation $\rho$ between the probability densities for the two modalities is also shown. 
above a chosen threshold were referred to as anisotropic regions, while those under this threshold were labeled isotropic. To highlight preferred orientation in each subimage, quiver plots were superimposed on anisotropic regions to give an orientation map. Histograms of $\theta$ for anisotropic regions (number of bins $=32$ ) and bar plots showing the isotropic and anisotropic region counts were also generated from these data. The $\theta$ histograms were further normalized to obtain orientation probability densities $P_{\mathrm{SHG}}(\theta)$ and $P_{\mathrm{SLIM}}(\theta)$. Figure 5 shows the comparable results obtained for a selected pair of segmented SLIM and SHGM images.

\subsection{Comparison Between Second-Harmonic Generation Microscopy and Spatial Light Interference Microscopy Signals}

Figure 6 shows the fiber orientation probability densities $P_{\text {SHG }}$ and $P_{\text {SLIM }}$ for three different cores, extracted from their respective SHGM and segmented SLIM images. As shown, the shapes of the density functions obtained from the two modalities are qualitatively similar for each of the cores. To obtain a quantitative measure of this similarity, the following procedure was used. The cross-correlation between $P_{\text {SHG }}$ and $P_{\text {SLIM }}$ was first obtained, and the circular lag corresponding to maximum cross-correlation was computed. The two densities were then shifted relative to one another by this lag, so any errors due to overall rotation between the two images are minimized. This alignment procedure is required for a fair comparison between the two densities because errors in registration of the images from the two modalities can cause one density to be slightly shifted with respect to the other. After alignment, the Pearson's correlation coefficient $\rho$ between the two densities was computed as a quantitative measure of their similarity. The Pearson's correlation measures the similarity of any two random variables $X$ and $Y$ and is defined as follows:

$\rho=\frac{E\left[\left(X-\mu_{x}\right)\left(Y-\mu_{y}\right)\right]}{\sigma_{x} \sigma_{y}}$.

In Eq. (7), the operator $E[]$ refers to the expected value, and $\mu$ and $\sigma$ are the mean and standard deviation, respectively, of the random variable in question. ${ }^{61}$ The correlation coefficient $\rho$ has values over the interval $[-1,1]$ with -1 referring to perfect negative correlation and 1 corresponding to perfect positive correlation. In our analysis, $X$ and $Y$ refer to the probability densities $P_{\text {SHG }}$ and $P_{\text {SLIM }}$.

The three cores in Fig. 6 belong to three different disease stages and, therefore, correspond to three different morphologies.

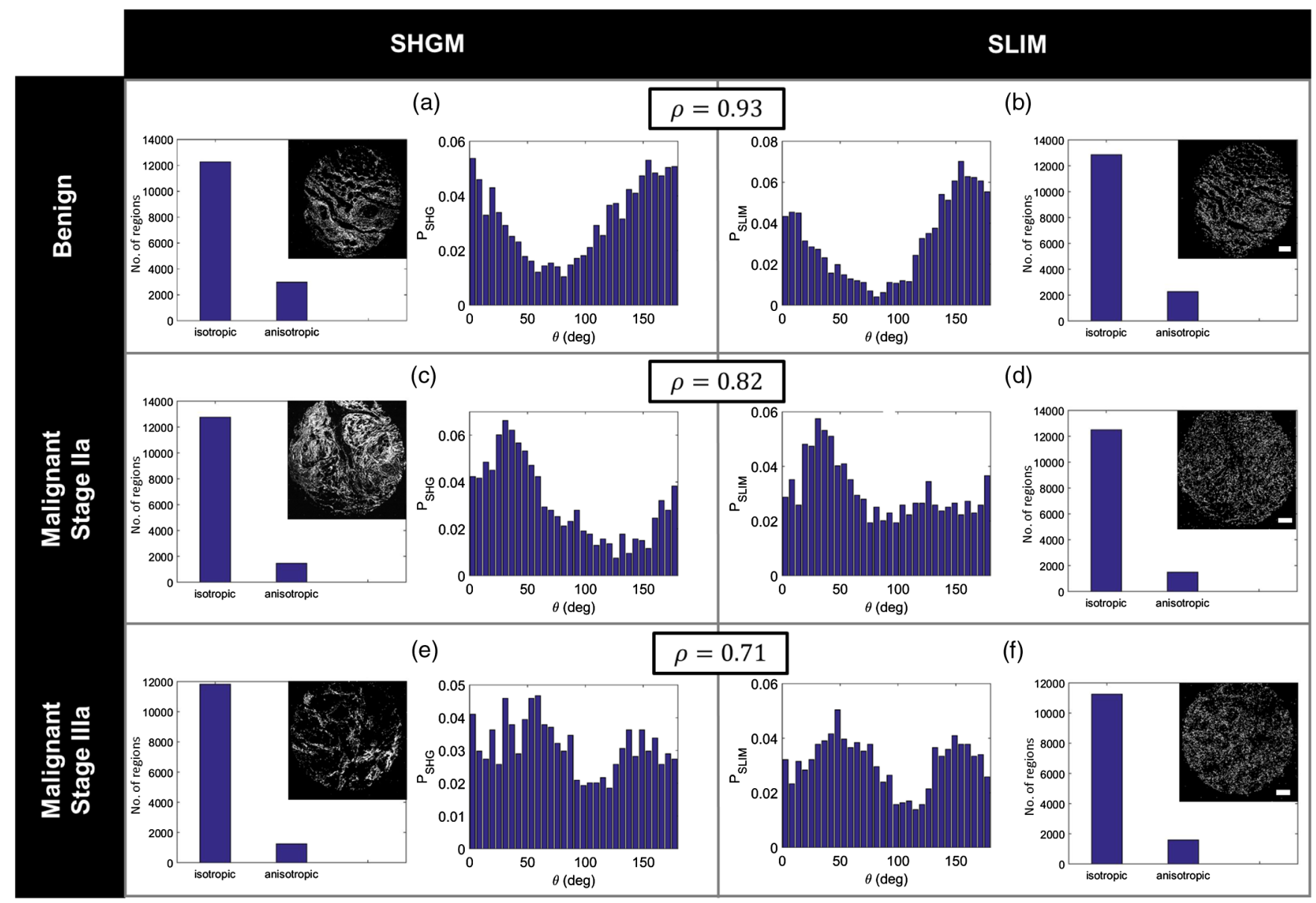

Fig. 6 Comparison between the fiber orientation probability densities and bar charts counting number of isotropic and anisotropic regions for three different cores: SHGM (left column) and SLIM (right column). This comparison is shown ( $a$ and $b$ ) for a benign core, (c and d) malignant stage lla core and (e and f) malignant stage Illa core. The similarity of $P_{\text {SHG }}$ and $P_{\text {SLIM }}$ is measured for each case using the Pearson's correlation coefficient $\rho$, as indicated. Scale bar: $200 \mu \mathrm{m}$. 
As shown, the benign core shows the highest positive correlation between $P_{\mathrm{SHG}}$ and $P_{\mathrm{SLIM}}$ with $\rho=0.93$, which decreases to 0.82 for the stage IIa malignant core. The lowest correlation is seen for the stage IIIa core, which was computed as 0.71 . This trend can be accounted for by the fact that a core from a patient at an advanced stage of disease is more likely to contain large amounts of epithelial tissue. A large proportion of epithelial tissue suppresses the SHG signal and results in greater image segmentation errors in SLIM images due to the smaller amount of collagen involved, resulting in lower agreement between the two. In addition to the probability densities, the bar charts showing the number of isotropic and anisotropic cells counted by the Fourier analysis procedure are also very similar for both imaging modalities, as demonstrated in Fig. 6 .

\section{Summary and Discussion}

Motivated by the relationship between $\chi^{(2)}$ and $\left[\chi^{(1)}\right]^{2}$ (Sec. 2), we presented experimental support for the hypothesis that measuring $\chi^{(1)}$ via phase-resolved imaging can provide $\chi^{(2)}$ information, similar to that obtained in SHGM. Specifically, we have shown that SHGM and segmented SLIM images measure quantitatively similar collagen fiber orientations in breast tissue. The conclusions are significant because collagen fiber alignment and orientation are potential markers for patient prognosis. While, in the past, SHGM has been the method of choice for measuring these biomarkers, our results demonstrate the potential of SLIM as a complementary method for this assessment. Clearly, compared to SHGM, SLIM lacks the specificity to collagen, as the phase image includes signals due to centrosymmetric molecules. As a result, a numerical postprocessing procedure is necessary to eliminate the isotropic structures from the SLIM image. However, these isotropic signals, mainly from epithelial contributions, can be used to delineate the glands and, potentially, tumor margins. These margins are difficult to detect in SHGM alone and other modalities are sometimes used (e.g., two-photon fluorescence microscopy). ${ }^{21,62}$

In comparing the optical setups of the two modalities, it is evident that SLIM benefits from common components, of much lower cost, compared to those needed in SHGM (e.g., halogen lamp versus femtosecond laser). Furthermore, due to the fullfield and continuous wave illumination, SLIM operates at much lower exposures and, thus, is nonperturbing to live cells and tissues. For example, SLIM imaging over multiple days without damage is possible. ${ }^{26}$ The acquisition rate of the SLIM system is $0.08 \mu \mathrm{s} / \mathrm{pixel}$, as compared to $7 \mu \mathrm{s} / \mathrm{pixel}$ for the state-of-the-art SHGM system used here. Of course, SLIM signals do not depend on phase matching, and, as such, the signals are always quantitatively related to the structure under investigation.

Before our method becomes adoptable to a clinical setting, further investigations on a large cohort of cases that are positive and negative for collagen biomarkers (such as TACS-3) are required. Since SLIM provides a quantitative map of tissue, it lends itself to the application of machine learning tools for automated analysis and classification. Development of computational tools for automated detection of biomarkers is crucial for future SLIM applications in high-throughput screening.

\section{Disclosures}

Gabriel Popescu has financial interest in Phi Optics, Inc., a company that develops quantitative phase imaging technologies.

\section{Acknowledgments}

We would like to thank Mikhail Kandel for developing the SLIM slide-scanning platform and Tan Nguyen for his insights regarding image processing. We would also like to thank Mayandi Sivaguru for the help with the SHGM imaging preparations. This work was supported by the National Science Foundation (Grant Nos. CBET-1040461 MRI, CBET-0939511 STC, DBI 1450962 EAGER, and IIP-1353368).

\section{References}

1. International Agency for Research on Cancer, "Cancer fact sheets: breast cancer," http://gco.iarc.fr/today/fact-sheets-cancers?cancer=15 \&type $=0 \&$ sex $=2$ (12 January 2016).

2. B. Lauby-Secretan et al., "Breast-cancer screening-viewpoint of the IARC Working Group," N. Engl. J. Med. 372, 2353-2358 (2015).

3. P. Taneja et al., "Classical and novel prognostic markers for breast cancer and their clinical significance," Clin. Med. Insights Oncol. 4, 15-34 (2010).

4. M. W. Conklin et al., "Aligned collagen is a prognostic signature for survival in human breast carcinoma," Am. J. Pathol. 178, 1221-1232 (2011).

5. S. Sridharan et al., "Prediction of prostate cancer recurrence using quantitative phase imaging," Sci. Rep. 5, 9976 (2015).

6. B. Weigelt, J. L. Peterse, and L. J. van't Veer, "Breast cancer metastasis: markers and models," Nat. Rev. Cancer 5, 591-602 (2005).

7. R. Kalluri and M. Zeisberg, "Fibroblasts in cancer," Nat. Rev. Cancer 6, 392-401 (2006).

8. L. M. Arendt et al., "Stroma in breast development and disease," Semin. Cell Dev. Biol. 21, 11-18 (2010).

9. S. O. Tamimi and A. Ahmed, "Stromal changes in invasive breast carcinoma: an ultrastructural study," J. Pathol. 153, 163-170 (1987).

10. D. Radisky, J. Muschler, and M. J. Bissell, "Order and disorder: the role of extracellular matrix in epithelial cancer," Cancer Invest. 20, 139-153 (2002).

11. A. Bergamaschi et al., "Extracellular matrix signature identifies breast cancer subgroups with different clinical outcome," J. Pathol. 214, 357-367 (2008).

12. M. Grossman et al., "Tumor cell invasion can be blocked by modulators of collagen fibril alignment that control assembly of the extracellular matrix," Cancer Res. 76, 4249-4258 (2016).

13. V. Ajeti et al., "Structural changes in mixed Col I/Col V collagen gels probed by SHG microscopy: implications for probing stromal alterations in human breast cancer," Biomed. Opt. Express 2, 2307-2316 (2011).

14. P. P. Provenzano et al., "Collagen reorganization at the tumor-stromal interface facilitates local invasion," BMC Med. 4, 1-15 (2006).

15. J. Locker and J. E. Segall, "Breast cancer: the matrix is the message," Am. J. Pathol. 178, 966-968 (2011).

16. X. Chen et al., "Second harmonic generation microscopy for quantitative analysis of collagen fibrillar structure," Nat. Protocols 7, 654-669 (2012).

17. R. Ambekar et al., "Quantifying collagen structure in breast biopsies using second-harmonic generation imaging," Biomed. Opt. Express 3, 2021-2035 (2012).

18. K. M. Riching et al., "3D collagen alignment limits protrusions to enhance breast cancer cell persistence,' Biophys. J. 107, 2546-2558 (2014).

19. J. S. Bredfeldt et al., "Computational segmentation of collagen fibers from second-harmonic generation images of breast cancer," $J$. Biomed. Opt. 19, 016007 (2014).

20. J. S. Bredfeldt et al., "Automated quantification of aligned collagen for human breast carcinoma prognosis," J. Pathol. Inf. 5, 28 (2014).

21. A. Zoumi, A. Yeh, and B. J. Tromberg, "Imaging cells and extracellular matrix in vivo by using second-harmonic generation and two-photon excited fluorescence," Proc. Natl. Acad. Sci. U. S. A. 99, 11014 11019 (2002).

22. G. Popescu, Quantitative Phase Imaging of Cells and Tissues, McGraw Hill, New York (2011).

23. B. Bhaduri et al., "Diffraction phase microscopy: principles and applications in materials and life sciences," Adv. Opt. Photonics 6, 57-119 (2014). 
24. Y. K. Park et al., "Refractive index maps and membrane dynamics of human red blood cells parasitized by Plasmodium falciparum," Proc. Natl. Acad. Sci. 105, 13730-13735 (2008).

25. Y. Park et al., "Measurement of red blood cell mechanics during morphological changes," Proc. Natl. Acad. Sci. 107, 6731-6736 (2010).

26. M. Mir et al., "Optical measurement of cycle-dependent cell growth," Proc. Natl. Acad. Sci. 108, 13124-13129 (2011).

27. T. Kim et al., "White-light diffraction tomography of unlabelled live cells," Nat. Photonics 8, 256-263 (2014).

28. R. Zhou et al., "Detecting $20 \mathrm{~nm}$ wide defects in large area nanopatterns using optical interferometric microscopy," Nano Lett. 13, 3716-3721 (2013).

29. B. Rappaz et al., "Comparative study of human erythrocytes by digital holographic microscopy, confocal microscopy, and impedance volume analyzer," Cytometry Part A 73A, 895-903 (2008).

30. W. Choi et al., "Tomographic phase microscopy," Nat. Methods 4, 717-719 (2007).

31. F. Merola et al., "Digital holography as a method for $3 \mathrm{D}$ imaging and estimating the biovolume of motile cells," Lab Chip 13, 4512-4516 (2013).

32. K. L. Cooper et al., "Multiple phases of chondrocyte enlargement underlie differences in skeletal proportions," Nature 495, 375-378 (2013).

33. K. Lee et al., "Quantitative phase imaging techniques for the study of cell pathophysiology: from principles to applications," Sensors $\mathbf{1 3}$, 4170-4191 (2013).

34. M. Mir et al., "Blood screening using diffraction phase cytometry," J. Biomed. Opt. 15, 027016 (2010).

35. M. Mir, K. Tangella, and G. Popescu, "Blood testing at the single cell level using quantitative phase and amplitude microscopy," Biomed. Opt. Express 2, 3259-3266 (2011).

36. B. Bhaduri et al., "Optical assay of erythrocyte function in banked blood," Sci. Rep. 4, 6211 (2014).

37. Y. Kim et al., "Profiling individual human red blood cells using common-path diffraction optical tomography," Sci. Rep. 4, 6659 (2014).

38. P. Memmolo et al., "3D morphometry of red blood cells by digital holography," Cytometry Part A 85, 1030-1036 (2014).

39. J. Yoon et al., "Label-free characterization of white blood cells by measuring 3D refractive index maps," Biomed. Opt. Express 6, 3865-3875 (2015).

40. N. T. Shaked et al., "Quantitative microscopy and nanoscopy of sickle red blood cells performed by wide field digital interferometry," J. Biomed. Opt. 16, 030506 (2011).

41. Z. Wang et al., "Tissue refractive index as marker of disease," J. Biomed. Opt. 16, 116017 (2011).

42. H. Majeed et al., "Breast cancer diagnosis using spatial light interference microscopy," J. Biomed. Opt. 20, 111210 (2015).

43. P. Wang et al., "Nanoscale nuclear architecture for cancer diagnosis beyond pathology via spatial-domain low-coherence quantitative phase microscopy," J. Biomed. Opt. 15, 066028 (2010).

44. S. Uttam et al., "Early prediction of cancer progression by depthresolved nanoscale mapping of nuclear architecture from unstained tissue specimens," Cancer Res. 75, 4718-4727 (2015).

45. Y. Bishitz et al., "Optical-mechanical signatures of cancer cells based on fluctuation profiles measured by interferometry," J. Biophotonics 7, 624-630 (2014).

46. B. Kemper et al., "Investigation of living pancreas tumor cells by digital holographic microscopy," J. Biomed. Opt. 11, 034005 (2006).

47. P. Wang et al., "Spatial-domain low-coherence quantitative phase microscopy for cancer diagnosis," Opt. Lett. 35, 2840-2842 (2010).

48. S. Sridharan et al., "Prediction of prostate cancer recurrence using quantitative phase imaging," Sci. Rep. 5, 9976 (2015).

49. S. Sridharan et al., "Prediction of prostate cancer recurrence using quantitative phase imaging: validation on a general population," Sci. Rep. 6, 33818 (2016).

50. H. Majeed et al., "Quantitative phase imaging for medical diagnosis," J. Biophotonics 10, 177-205 (2017).

51. Z. Wang et al., "Spatial light interference microscopy (SLIM)," Opt. Express 19, 1016-1026 (2011).
52. Z. Wang et al., "Spatial light interference tomography (SLIT)," Opt. Express 19, 19907-19918 (2011).

53. R. W. Boyd, Nonlinear Optics, Academic Press, Massachusetts (2008).

54. T. Santosh et al., "Determination of the second-order nonlinear susceptibility elements of a single nanoparticle using coherent optical microscopy," J. Phys. B 44, 015401 (2011).

55. R. A. R. Rao, M. R. Mehta, and K. C. Toussaint, "Fourier transformsecond-harmonic generation imaging of biological tissues," Opt. Express 17, 14534-14542 (2009).

56. C. Okoro and J. K. C. Toussaint, "Experimental demonstration of twophoton Mueller matrix second-harmonic generation microscopy," J. Biomed. Opt. 21, 016011 (2016).

57. T. Leung and J. Malik, "Representing and recognizing the visual appearance of materials using three-dimensional textons," Int. J. Comput. Vision 43, 29-44 (2001).

58. M. Varma and A. Zisserman, "A statistical approach to texture classification from single images," Int. J. Comput. Vision 62, 61-81 (2005).

59. N. Otsu, "A threshold selection method from gray level histograms," IEEE Trans. Syst. Man Cybern. 9, 62-66 (1979).

60. Visual Geometry Group, University of Oxford, "Matlab script for generating our implementation of the L-M filter bank," Texture classification, http://www.robots.ox.ac.uk/ vgg/research/texclass/code/ makeLMfilters.m.

61. M. H. DeGroot and M. J. Schervish, Probability and Statistics, Addison-Wesley, Boston (2012).

62. S. W. Perry, R. M. Burke, and E. B. Brown, "Two-photon and second harmonic microscopy in clinical and translational cancer research," Ann. Biomed. Eng. 40, 277-291 (2012).

Hassaan Majeed is a PhD candidate in the Bioengineering Department at the University of Illinois at Urbana-Champaign. He is working in the Quantitative Light Imaging (QLI) Lab at the Beckman Institute of Advanced Science and Technology; his research is focused on using quantitative phase imaging for developing new methods for breast cancer diagnosis as well as looking for indicators of prognosis

Chukwuemeka Okoro is a doctoral candidate in the Department of Electrical and Computer Engineering at the University of Illinois at Urbana Champaign (UIUC). He has his master's degree in electrical engineering from UIUC and his bachelor's degree in electrical engineering from University of Lagos, Nigeria. His current work involves developing quantitative second harmonic generation imaging techniques as a tool for assessing biological tissues.

André Kajdacsy-Balla is a professor and director of transdisciplinary pathology at the University of Illinois at Chicago. His clinical focus is anatomic pathology with special interest in gynecologic pathology and prostate cancer. His research interests are in the areas of tissue banking, tissue microarrays, application of molecular techniques to tissue pathology, prostate cancer clinical outcomes prediction methods, and the effect of environmental agents on prostate cancer progression and metastasis.

Kimani C. Toussaint is an associate professor in the Department of Mechanical Science and Engineering and an affiliate in the Departments of Electrical and Computer Engineering, and Bioengineering at the University of Illinois at Urbana-Champaign. He directs an interdisciplinary lab that focuses on developing optical techniques for quantitatively imaging collagen-based tissues and investigating the properties of plasmonic nanostructures for control of near-field optical forces. He is a senior member in SPIE, OSA, and IEEE

Gabriel Popescu is an associate professor in electrical and computer engineering at the University of Illinois at Urbana-Champaign. His research is focused on biomedical optics, with special emphasis on interferometric label-free imaging. He has published a book on QPI, authored 130 journal publications and 1850 conference presentations, obtained 32 patents, and given 150 invited/keynote/plenary talks. He founded Phi Optics, Inc., a startup company that commercializes quantitative phase imaging technology. He is a fellow of OSA and SPIE. 